\title{
Optimizing Network Performance In Replicated Hosting
}

\author{
Ningning $\mathrm{Hu}^{\dagger}$ \\ hnn@cs.cmu.edu \\ Oliver Spatscheck ${ }^{\S}$ \\ Jia Wang ${ }^{\S}$ \\ jiawang@research.att.com \\ Peter Steenkiste ${ }^{\dagger}$ \\ prs@cs.cmu.edu \\ † Carnegie Mellon University, Pittsburgh, PA 15213, USA \\ $\S$ AT\&T Labs - Research, Florham Park, NJ 07932, USA
}

\begin{abstract}
Most important commercial Web sites maintain multiple replicas of their server infrastructure to increase both reliability and performance. In this paper, we study how many replicas should be used and where they should be placed in order to improve client network performance, including both the latency (e.g., round-trip time) between clients and the replicas, and the bandwidth performance between them. This study is based on a large scale measurement study from an 18-node infrastructure, which reveals for the first time the distribution of today's Internet end-user access bandwidth. For example, we find that $50 \%$ of end users have access bandwidth less than 4.2Mbps. Using a greedy algorithm, we show that the first five replicas dominate latency optimization in our measurement infrastructure, while the first two replicas dominate bandwidth optimization. We also found that geographic diversity does not help as much for bandwidth optimization as it does for latency. To determine the proper trade-off between latency and bandwidth, we use a simplified TCP model to show that, when content size is less than 10KB, the deployment should focus on optimizing latency, while for content sizes larger than $1 M B$, the deployment should focus on optimizing bandwidth.
\end{abstract}

\section{Introduction}

It is increasingly common for content providers to use replication to increase the reliability and performance of their server infrastructure. Some content providers use commercial Content Distribution Networks (CDNs) [22] to achieve this goal, while others replicate their server infrastructure in multiple locations. Most large content providers use both techniques depending on the type and the value of the content delivered. In this paper, we focus on the latter method, i.e., replicating the whole server infrastructure. This technique has two advantages over CDNs. First, replicating the entire server infrastructure (we will call each replicated server a replica) at multiple sites not only al- lows the content to be delivered from multiple locations, but also allows the content provider to perform more complicated tasks such as securing e-commerce transactions which require real time database access. Second, unlike CDNs which require a master server as the content origin, replicas allow the content provider to operate seamlessly if one of its replicas is not available.

In this paper we focus on how replication can improve performance. The most direct metric for optimizing content replications is content download time. However, it is impossible to measure download time from a collection of possible replicas to every client on the Internet. To address this problem, multiple solutions have been proposed including the use of geographic location [19], network mapping services [7], and network hops and latency [6, 23, 4, 18]. In this paper, we estimate the download time for objects of various sizes by measuring the round-trip time (RTT) and the available bandwidth to each prefix using Pathneck [8]. In our optimization, we consider both the overall performance and the performance of the $20 \%$ worst destinations.

Our contributions are four-fold. First, we show that most hosts access the Internet through relatively low bandwidth links and for these hosts, replica selection based on bandwidth has no benefit. Second, for well-provisioned hosts, the first two replicas provide most of the improvement that can be gained from bandwidth optimization, while we will need five replicas for RTT optimization. Third, we demonstrate that replica placement to optimize bandwidth does not follow a clear geographic pattern, while RTT optimization does. Fourth, we use a simple TCP data transmission model to show that when the content size is less than $10 \mathrm{~KB}$, the replica deployment should minimize RTT; when the content size is larger than $1 \mathrm{MB}$, the deployment should maximize bandwidth.

The remainder of the paper is structured as follows. We describe our measurement methodology in Section 2, and summarize our measurement results in Section 3. We then present the results of both bandwidth and RTT optimization under different application scenarios in Section 4. Section 5 uses a simplified TCP model to evaluate the trade-off be- 


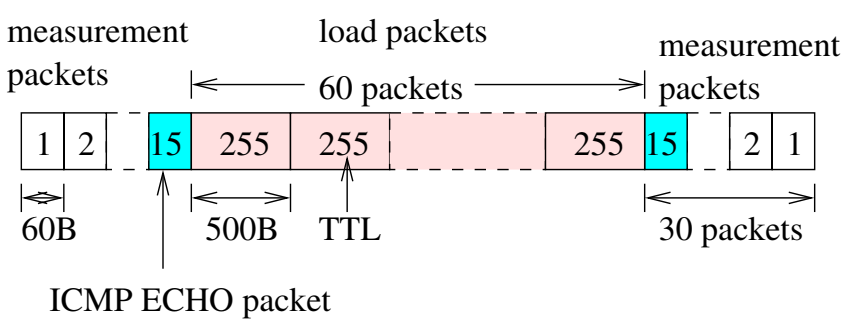

\section{Figure 1. Probing packet train of the modified Pathneck}

tween RTT and bandwidth optimization. Finally, we present the related work in Section 6 and conclude in Section 7.

\section{Methodology}

In this section, we first briefly review the measurement tool that we used. We then describe our measurement infrastructure followed by a discussion of the implications of our methodology.

\subsection{Measurement Tool-Pathneck}

Our measurement data was obtained using Pathneck [8], which is an active probing tool that allows end users to efficiently and accurately locate bottleneck links on the Internet. Pathneck is based on a novel way of constructing a probing packet train-Recursive Packet Train, which combines load packets and measurement packets. The load packets interleave with background traffic, which affects the length of the Pathneck train. The change in train length provides information about the available bandwidth on a link. The measurement packets have carefully controlled TTL values, so that the ICMP packets generated when packets expire can be used by the source to estimate the length of the train at every hop in the network path. Pathneck can estimate the bottleneck location and an upperbound on the available bandwidth on the bottleneck link (and thus the path). Similar to traceroute, it also measures the route and the RTT. Details on Pathneck can be found elsewhere [8].

A problem with the original Pathneck tool is that it cannot measure the last hop of Internet paths, which are good candidate bottlenecks. For our measurement, we modified the Pathneck tool to alleviate this problem. The idea ${ }^{1}$ is to use ICMP ECHO packets instead of UDP packets as the measurement packets for the last hop. For example, the packet train in Figure 1 is used to measure a 15-hop path. If the destination replies to the ICMP ECHO packet, we can

\footnotetext{
${ }^{1}$ It was originally suggested by Tom Killlian from AT\&T LabsResearch.
}

Table 1. Measurement source nodes

\begin{tabular}{|c|c||c|c||c|c|}
\hline ID & Location & ID & Location & ID & Location \\
\hline \hline$S 01$ & US-NE & $S 07$ & US-SW & $S 13$ & US-NM \\
\hline$S 02$ & US-SM & $S 08$ & US-MM & $S 14$ & US-NE \\
\hline$S 03$ & US-SW & $S 09$ & US-NE & $S 15$ & Europe \\
\hline$S 04$ & US-MW & $S 10$ & US-NW & $S 16$ & Europe \\
\hline$S 05$ & US-SM & $S 11$ & US-NM & $S 17$ & Europe \\
\hline$S 06$ & US-SE & $S 12$ & US-NE & $S 18$ & East-Asia \\
\hline
\end{tabular}

NE: northeast, NW: northwest, SE: southeast, SW: southwest

ME: middle-east, MW: middle-west, MM: middle-middle

obtain the gap value on the last hop. In order to know where the ICMP packets should be inserted in the probing packet train, Pathneck first uses traceroute to get the path length. Note that this Pathneck modification works only for Internet destinations that reply to ICMP ECHO packets.

\subsection{Measurement Infrastructure}

In our measurement, we used 18 measurement sources (Table 1), 14 of which are in the US, 3 are in Europe, and 1 is in East-Asia. All the sources directly connect to a large Tier-1 ISP (referred as $X$ ) via 100Mbps Ethernet links. In this paper, we consider these 18 sources as the candidate locations that an application can use to replicate its server infrastructure. In the rest of this paper, we also refer to these sources as replicas.

The measurement destinations are selected as follows. Our goal is to cover as many diverse locations on the Internet as possible. We select one IP address as the measurement destination from each of the 164,130 prefixes extracted from a BGP table. Ideally, the destination should correspond to an online host that replies to ping packets. However it is difficult to identify online hosts without probing them. In our study, we partially alleviate this problem by picking IP addresses from three pools of existing data sets collected by the ISP: Netflow traces, client IP addresses of some Web sites, and the IP addresses of a large set of local DNS servers. That is, for each prefix, whenever possible, we use one of the IP addresses from those three sources in that prefix; otherwise, we randomly pick an IP address from that prefix. In this way, we are able to find reachable destination in 67,271 of the total 164,130 prefixes.

We collected Pathneck data from the 18 sources to the 164,130 selected destinations from August 28, 2004 to September 30, 2004. Note that for those unreachable destinations, we only have partial path information. Ideally, the partial paths would connect the measurement sources to a common join node that is the last traced hop from each source, so the join node stands in for the destination in a consistent way. Unfortunately, such a join node may not 
exist, either because there may not be a node shared by all the 18 measurement sources to a given destination or the shared hop may not be the last traced hop. For this reason, we relax the constraints for the join node: the join node only needs to be shared by 14 measurement sources, and it need only be within three hops from the last traced hop. Here "14" is simply a reasonably large number that we choose. This allows us to make the best of the available data while still having comparable data. Specifically, this allows us to include over $141 \mathrm{~K}$ destinations ( $86 \%$ of the total) in our analysis, among which $47 \%$ are real destinations, and $53 \%$ are join nodes. We refer to these $141 \mathrm{~K}$ destinations as valid destinations.

\subsection{Applicability to Other ISPs}

Since all our measurement sources are connected to the same ISP $X$, our analysis results could easily be specific to ISP $X$. However, we believe that the results are more broadly applicable. The intuition is that the widely used "hot-potato" routing approach forces packets to quickly enter other ISPs' networks if they need to traverse those networks to reach their destinations. For those packets, the time spent in ISP $X$ is very small. Since ISP $X$ is very well engineered and its links rarely appear as bottlenecks in our measurements, ISP $X$ is largely "transparent" in these cases and our measurement sources can be considered as reasonable vantage points for those ISPs.

To validate this intuition, we pick 7 top Tier-1 ISPs peering with ISP $X$ from Keynote [3]; 5 of the ISPs are in the US and 2 are in Europe. $64 \%$ of all the paths enter these ISPs directly from ISP $X$. Figure 2 shows the time spent within $X$ (referred as exit time) by the packets on those paths. The top graph shows the median (middle circle), 10th percentile (bottom bar, generally overlapped with the circle), and 90th percentile (top bar) values of the exit time for all the measurement sources. It is not surprising that $S 15, S 16, S 17$, and $S 18$ have large exit times since they are in Europe or East-Asia, and have to use the trans-atlantic/pacific links to reach the peering points. The bottom graph shows the median, 70th percentile, and 90th percentile values of the exit time distributions for the 14 measurement sources located in the US. For each measurement source, over $70 \%$ of the paths have exit times less than $15 \mathrm{~ms}$; most of them are less than $10 \mathrm{~ms}$. The small exit times confirm our claim that our measurement setup and the corresponding analysis are also useful for the scenario where the replicas are located at sources connected to other major Tier-1 ISPs, and the scenario where the whole replicated service is located at a source which multihomes to all major Tier-1 ISPs.
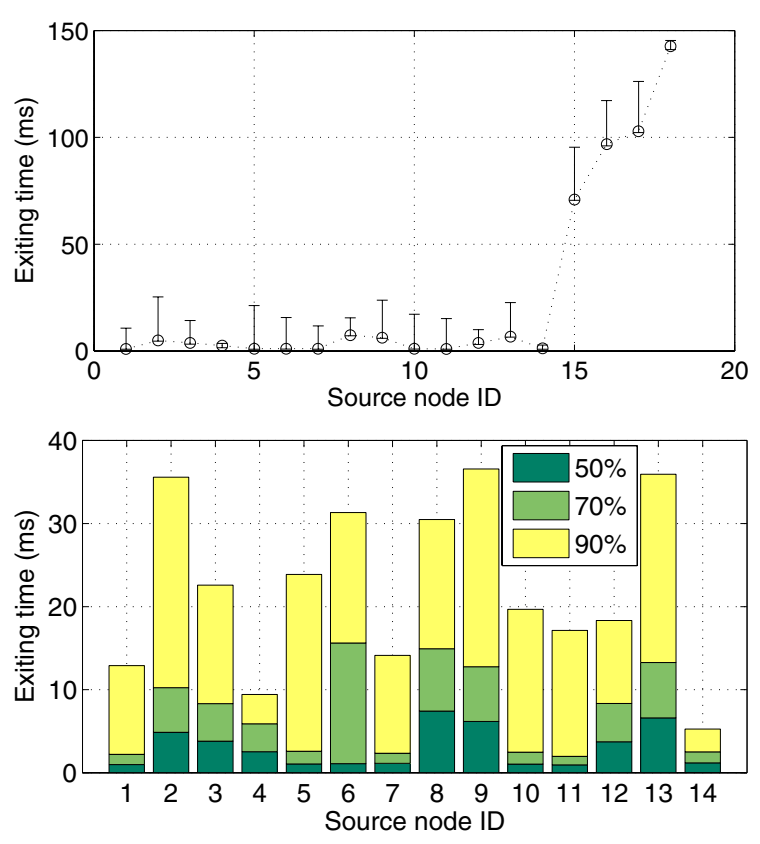

Figure 2. The exit times to neighbor ISPs.

\section{Performance of Individual Replica}

In this section, we look at the RTT and bandwidth performance from each individual replica to all destinations to understand their potential in improving RTT and bandwidth performance.

\subsection{RTT Performance}

Figure 3 shows the cumulative distribution (CDF) of RTT measurements from individual replicas. For the sake of clarity, we only plot the measurements for three replicas: $S 01, S 17$, and $S 18$, which are in the United States, Europe, and East Asia, respectively. The curve labeled with "best" corresponds to the optimal performance using all 18 replicas, i.e., the smallest RTT from all 18 replicas. We can see that RTT can be significantly improved by using multiple replicas. In addition, the performance of using a single replica varies depending on its location. These results show how the physical location of replicas plays an important role in RTT optimization.

\subsection{Bandwidth Performance}

Figure 4 illustrates the cumulative distributions of bandwidth measurements. Because Pathneck only provides an upper-bound of path available bandwidth, the bandwidth distributions shown in Figure 4 are upper-bounds for the actual bandwidth distributions. Compared with Figure 3, we 


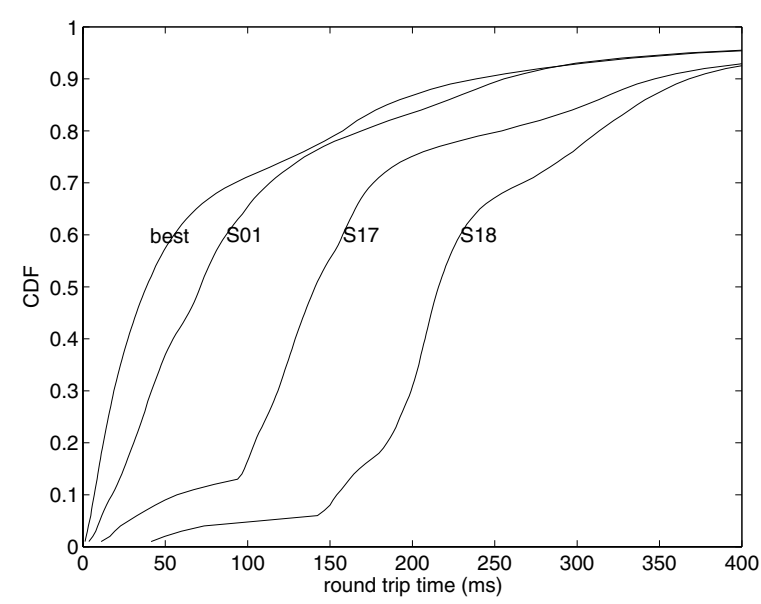

\section{Figure 3. RTT performances from individual replicas}

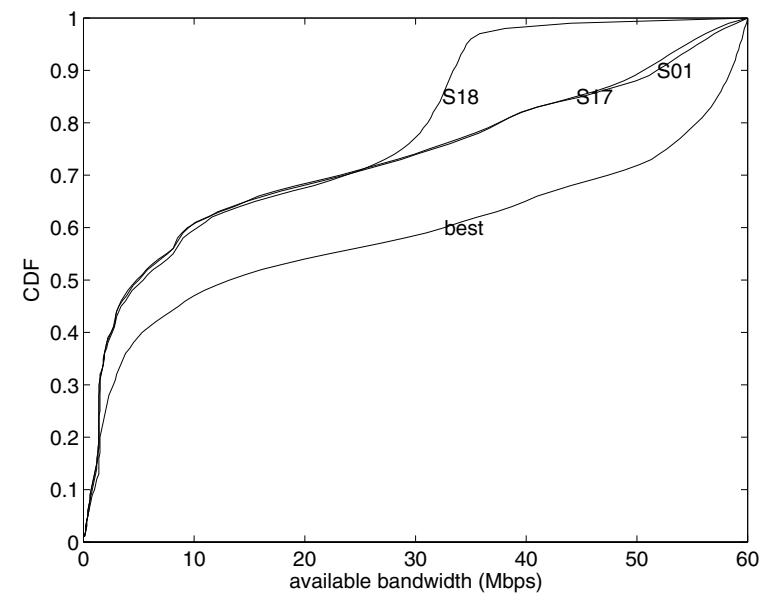

Figure 4. Bandwidth performances from individual replicas

see that the "best" curve also shows an improvement over those for individual replicas, but the difference among individual replicas is less pronounced. This implies that geographic diversity may not play as important a role for bandwidth optimization.

The above observation from Figure 4 is mainly due to the fact that bottlenecks on most of the measured paths are on the last few hops, and different replicas often share bottlenecks. Figure 5 plots the distribution of bottlenecks for the 67,271 destinations of which the last hop can be measured by Pathneck. The $x$-axis is the hop distance from bottleneck hop to destination, the $y$-axis is the percentage of destinations in each category. We observe that $75 \%$ of destinations

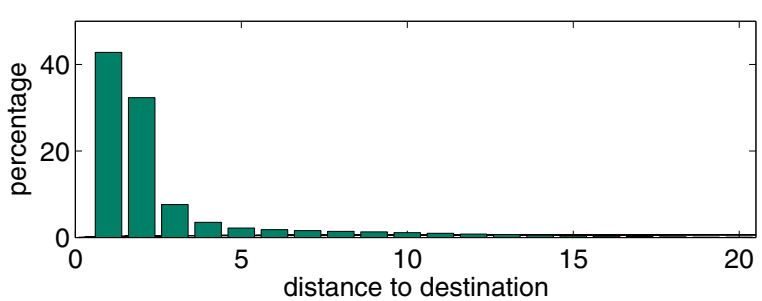

Figure 5. Bottleneck location distribution

have bottleneck on the last two hops, and $42.7 \%$ on the last hop.

Replicas can improve bandwidth performance if paths from different replicas to a destination have different bottlenecks and have different bottleneck bandwidths. Unfortunately, we find that in most cases the replicas either share bottlenecks, or have different bottleneck locations but the bottleneck links have very similar bandwidths. This leaves us little opportunity to improve bandwidth performance using replicas for end users. In some cases, we do observe different bottleneck bandwidths from different replicas, but that is because of traffic-load changes instead of bottleneck location changes. That is, the difference between the "best" curve in Figure 4 and those from individual replicas is mainly due to traffic-load changes.

To filter out the impact of traffic-load changes on bandwidth distribution, we select a bandwidth measurement for each destination that is most representative among those from all 18 replicas. This is done by splitting the 18 bandwidth measurements into several groups, and by taking the median value of the largest group as the representative bandwidth. The group is defined as the follows. Let $G$ be a group, and $x$ be a bandwidth value, $x \in G$ iff $\exists y, y \in G,|(x-y) / y|<0.3$. Figure 6 plots the distribution of the representative available bandwidths for the 67,271 destinations for which Pathneck can measure the last hop. To the best of our knowledge, this is the first result on the distribution of Internet-scale end-user access bandwidth. We observe that $40 \%$ of destinations have bottleneck bandwidth less than $2.2 \mathrm{Mbps}, 50 \%$ are less than $4.2 \mathrm{Mbps}$, and $63 \%$ are less than $10 \mathrm{Mbps}$. These results show that small capacity links still dominate Internet end-user connections. In such cases, it is difficult to use replicas to improve bandwidth performance because bottlenecks are very likely due to small-capacity last-mile links such as DSL, cable-modem, and dial-up links.

For the destinations with bottleneck bandwidth larger than $10 \mathrm{Mbps}$, the bottleneck bandwidth is almost uniformly distribute in the range of $[10 \mathrm{Mbps}, 50 \mathrm{Mbps}]^{2}$. For these

\footnotetext{
${ }^{2}$ The distribution curve in Figure 6 ends at $55.2 \mathrm{Mbps}$. This is a bias introduced by our measurement infrastructure. The sending rates from our
} 


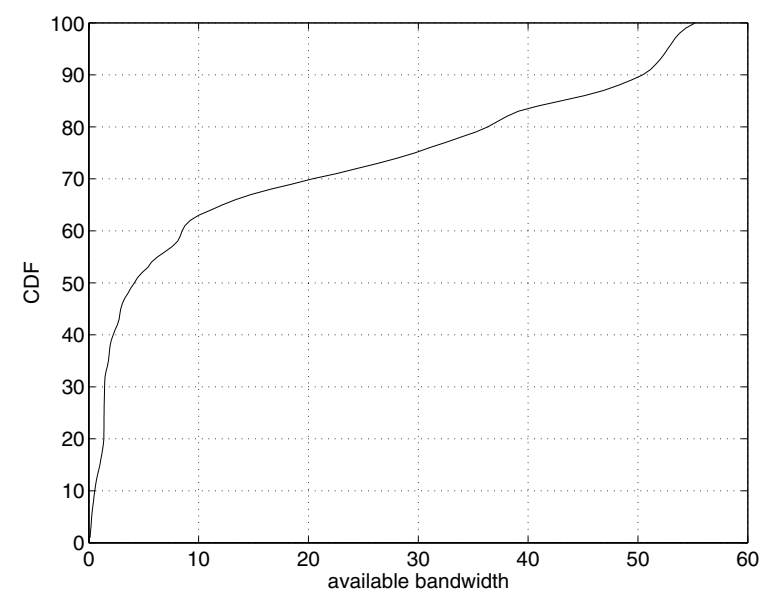

Figure 6. Distribution of Internet end-user access bandwidth

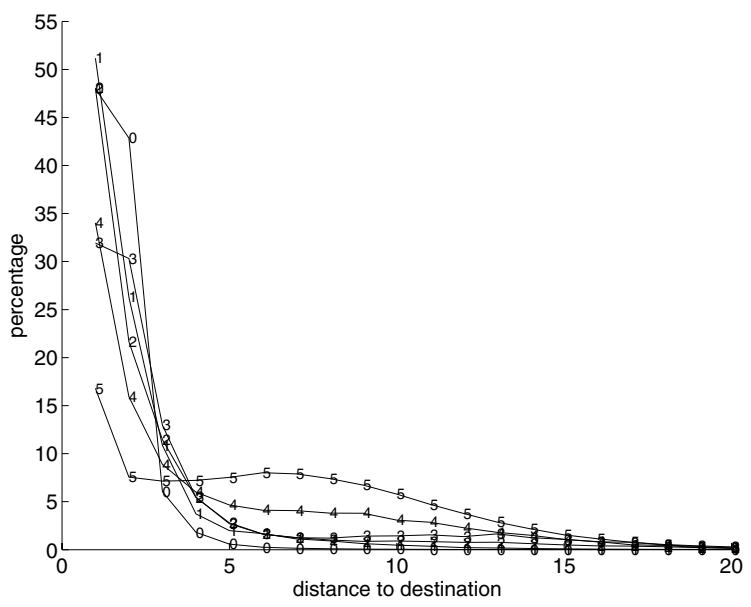

Figure 7. Bottleneck distribution for destinations with different available bandwidth

destinations, high-bandwidth bottlenecks are more likely to be determined by link load instead of by link capacity, and bottlenecks more frequently appear in the middle of paths. This observation is illustrated in Figure 7, where we split the 67,271 destinations used in Figure 5 into different groups based on bottleneck bandwidth, and plot the distribution of bottleneck locations for each group. The curve marked with " $i$ " represents the group of destinations which have bottleneck bandwidth in the range of $[i * 10 \mathrm{Mbps},(i+1) * 10 \mathrm{Mbps})$. We can see that while groups $0 \sim 3$ have distributions very similar with that

replicas are generally less than $60 \mathrm{Mbps}$, which determines the maximum bottleneck bandwidth that we can detect. shown in Figure 5, groups 4 and 5 are clearly different. For example, $62 \%$ of destinations in group 5 have bottlenecks that are over 4 hops away from the destinations, where different replicas have a higher probability of having different routes and thus different bottlenecks. Section 4.3 discusses how to make use of this opportunity to avoid bottlenecks by using different replicas for different destinations.

\section{Optimizing RTT and Bandwidth}

We have seen that replicas have the potential to improve RTT performance for most destinations, and to improve bandwidth performance for well provisioned destinations. In this section, we present an algorithm that allows us to select replicas to optimize client performance. We then use this algorithm to study how to improve RTT and bandwidth using our measurement infrastructure.

\subsection{The Greedy Algorithm}

To optimize client performance using replicas, we need to know how many replicas we should use and where they should be deployed. The goal is that the selected set of replicas should have performance very close to that achieved by using all replicas. A naive way is to consider all the possible combinations of replicates when selecting the optimal one. Given that there are $2^{18}-1$ (i.e., 262,143) different combinations for 18 replicas, and each replica measures over $160 \mathrm{~K}$ destinations, the time of evaluating all combinations is prohibitively high. Therefore, we use a greedy algorithm, which is based on a similar idea as the greedy algorithm used in Qiu et.al. [21]. This algorithm only needs polynomial processing time, but can find a sub-optimal solution very close to the optimal one. We now explain how to apply this algorithm on the data sets we have.

The intuition behind the greedy algorithm is to always pick the best replica among the available replicas. For example, in Figure 3, $S 01$ is better than $S 17$ and $S 18$ for RTT optimization, so $S 01$ should be selected before the other two. In this algorithm, the "best" is quantified using a metric called marginal utility. In the following, we explain how this metric is computed.

We use RTT optimization as the example. Assume that at some point, some replicas have already been selected. The best RTT from among the selected replica to each destination is given by $\left\{r t t_{i} \mid 0 \leq i<N\right\}$, where $N \approx 160 K$ is the number of destinations, and $r t t_{i}$ is the smallest RTT that is measured by one of the replicas already selected to destination $i$. Let $\left\{\operatorname{srtt}_{i} \mid 0 \leq i<N\right\}$ be the sorted version of $\left\{r t t_{i}\right\}$. We can now compute $r t t_{-}$sum as:

$$
r t t_{-} s u m=\sum_{k=0}^{99} s r t t_{i n d e x(k)}
$$


where

$$
\operatorname{index}(k)=\frac{N \times(k+1)}{101}-1
$$

Intuitively, $\left\{\operatorname{srtt}_{i} \mid 0 \leq i<N\right\}$ corresponds to the $x$ axis values of the points on the CDF curves in Figure 3, and rtt_sum corresponds to the area enclosed by the CDF curve, the left $y$-axis, and the top $x$-axis.

There are two details that need to be explained in the above computation. First, we cannot simply add all the individual measurement when calculating rtt_sum. This is because by definition, a joint node is not necessarily shared by all the measurement sources, so introducing a new replica could bring in measurements to new destinations, thus changing the value of $N$. Therefore, we cannot simply add all $r t t_{i}$ since the data sets would be incomparable. In our analysis, we add 100 values that are evenly distributed on the CDF curve. Here, the number "100" is empirically selected as a reasonably large value to split the curve. Second, we split the curve into 101 segments using 100 splitting points, and only use the values of these 100 splitting points. That is, we do not use the two end values $-s r t t_{0}$ and $s r t t_{N-1}$, whose small/large values are very probably due to measurement error.

Suppose a new replica $A$ is added, many $r t t_{i}$ can change,

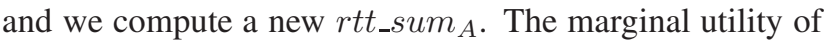
$A$ is then computed as:

$$
\text { marginal_utility }=\frac{\left|r t t_{-} s u m-r t t \_s u m_{A}\right|}{r t t \_s u m}
$$

With this definition, the replica selected in each step is the one that has the largest marginal utility. For the first step, the replica selected is simply the one that has the smallest rtt_sum. In the end, the algorithm generates a replica selection sequence:

$$
v_{1}, v_{2}, \ldots, v_{18}
$$

where $v_{i} \in\{S 01, S 02, \ldots, S 18\}$. To select $m(<18)$ replicas, we can simply use the first $m$ replicas in this sequence.

For bandwidth optimization, the greedy algorithm works in a similar way except $b w_{i}$ should be the largest bandwidth measured from one of the selected replicas. This greedy algorithm has polynomial processing time, but only gives a sub-optimal solution. In the following section, we will show that the greedy algorithm produces solutions that are very close to optimal.

\subsection{RTT Optimization}

As demonstrated in Section 3.1, RTT performance is closely related with replica location. In this section, we look at the number of replicas needed to improve RTT.

Figure 8 shows the results of RTT optimization using our greedy algorithm on all valid destinations. The top figure
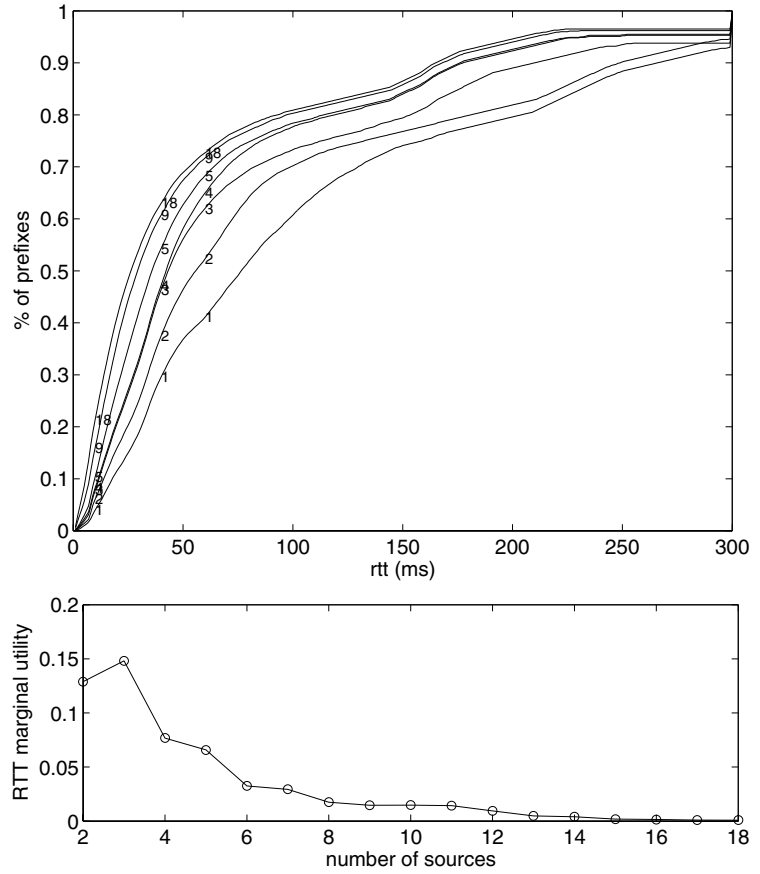

Figure 8. RTT optimization using greedy algorithm.

plots the cumulative distribution of RTT with different numbers (marked on the curves) of replicas. For clarity, we only plot the results when RTT $<300 \mathrm{~ms}$, though some RTTs are as large as 3 seconds. The bottom figure plots the marginal utility for each new replica added ${ }^{3}$. Clearly, the first five replicas improve the performance most significantly. The marginal utilities from additional replicas are all less than $5 \%$.

In addition, it is interesting to note the different patterns of RTT improvement with each of the first five replicas added. As we can see from the top figure, adding the second replica improves RTT over a very large range $[30 \mathrm{~ms}, 300 \mathrm{~ms}]$, though the improvement in the range $[50 \mathrm{~ms}, 100 \mathrm{~ms}]$ is the largest. Adding the third replica mainly improves RTT in the ranges [30 $\mathrm{ms}, 100 \mathrm{~ms}$ ] and $[150 \mathrm{~ms}, 250 \mathrm{~ms}]$; adding the fourth replica improves RTT in the range $[70 \mathrm{~ms}, 200 \mathrm{~ms}$ ], but with a slightly lower magnitude; and adding the fifth replica improves RTT in the range $[10 \mathrm{~ms}, 50 \mathrm{~ms}]$, with a further reduced magnitude.

Not surprisingly, these differences are mainly due to the different geographic locations of the replicas. As listed in

\footnotetext{
${ }^{3}$ This curve starts from the second replica because the first one is selected using a different method. Also the marginal utility of the third selected source is larger than that of the second. This is possible because in the marginal-utility formula the rtt_sum used to select the third source is less than that used to select the second.
} 
the row labeled "rtt-all" in Table 2-the first five are from US east-coast, Europe, US west-coast, East Asia, and US middle, respectively, which are consistent with common intuition. If we consider these geographic locations together with the delay range that is most improved by each replica, we can gain a better understanding of how replicas contribute to the performance improvement for destinations in different physical locations.

The greedy algorithm theoretically only provides suboptimal solutions. To understand how well it approximates the optimal solution, we compare the results in Figure 8 with the best solution obtained using a brute-force search. Obviously, brute-force search can not explore all the possible combinations of the 18 replicas, as explained before. However, we have seen that the first five replicas dominate performance gains. Given the computing resources we have, we can go through all possible four-replica combinations in five hours. Therefore, we compute the rtt_sum values for all four-replica combinations, and select the best one to compare with the first four replicas selected by our greedy algorithm. Using this method, we find that the solution obtained using our greedy algorithm for RTT optimization is the 7th best among all of the 3,060 four-replica combinations, and it is only $0.1 \%$ worse than the best solution ( $S 04 S 14 S 16 S 18$ ). Such small difference shows that the greedy algorithm indeed finds a solution close to the optimal one.

\subsection{Bandwidth Optimization}

Section 3.2 shows that replicas have higher probability to improve bandwidth performance for well provisioned destinations, since they have higher probability to experience different bottlenecks for such destinations. In this section, we use the greedy algorithm to study how to make use of this opportunity. We look at two cases. In the first case, we only use the 23,447 destinations where Pathneck can measure the last hop, and the bottleneck bandwidth is higher than $40 \mathrm{Mbps}$. In the second case, we include all valid destinations.

The results of the first case study are shown in Figure 9. It shows the cumulative distribution of path bandwidth upper-bounds with varying numbers of replicas. We can see that the bandwidth improvement from replicas is indeed significant. For example, with a single replica, there are only $26 \%$ paths that have bandwidth higher than $54 \mathrm{Mbps}$, while with all 18 replicas, the percentage increases to $65 \%$.

An obvious problem for the first case study is that it only covers around $16 \%$ of the paths that we measured, thus the results could be biased. To do a more general study, we apply the greedy algorithm on all the valid destinations, but exclude last-mile bottlenecks. In other words, we do

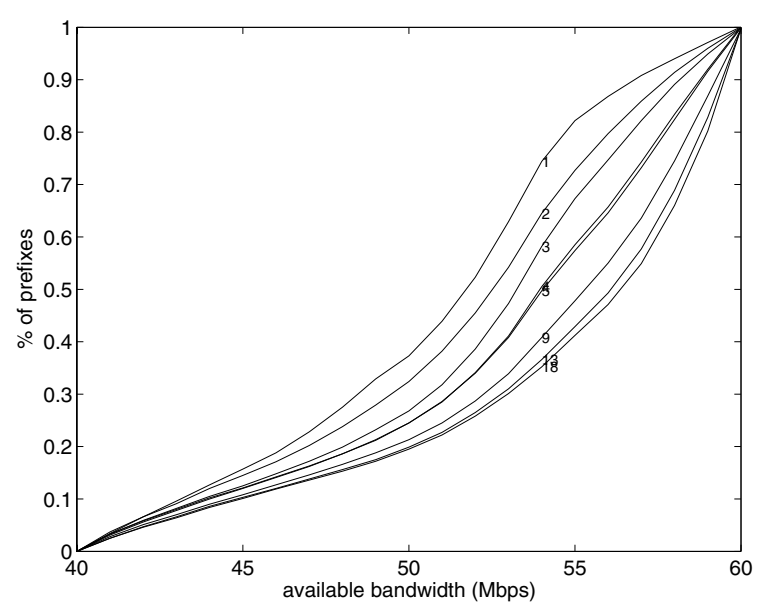

Figure 9. Bandwidth optimization for well provisioned destinations.
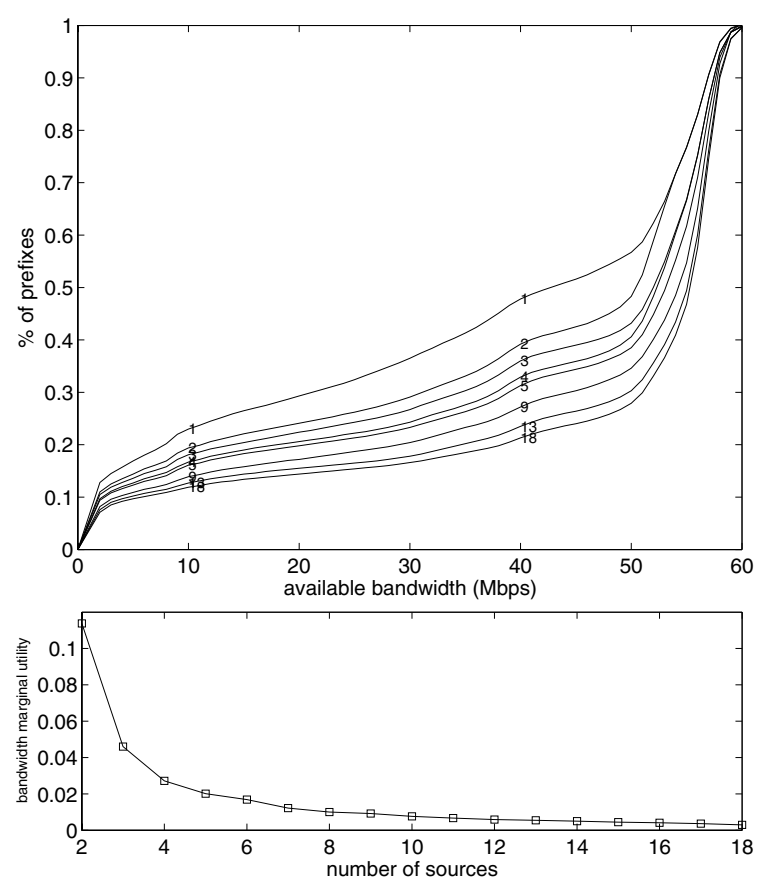

Figure 10. Bandwidth optimization for all valid destinations.

a "what if" experiment to study what would happen if lastmile links were upgraded. That is, for those destinations where Pathneck can measure the last hop, we remove the last two hops; this is based on the observation in Figure 5 that $75 \%$ paths have bottlenecks on the last two hops. For the others, we use the raw measurement results. Figure 10 
Table 2. The replica selection sequence (with location code) determined by the greedy algorithm

\begin{tabular}{|c|rrrrrrrrrrrrrrrrr|l|l|l|}
\hline ID & \multicolumn{10}{|l|}{ Replica selection sequence } & Reference \\
\hline rtt-all & S14 & S17 & S04 & S18 & S11 & S05 & S16 & S01 & S06 & S07 & S15 & S10 & S08 & S12 & S02 & S03 & S13 & S09 & Section 4.2 \\
& NE & Eu & MW & AS & NM & SM & Eu & NE & SE & SW & Eu & NW & MM & NE & SM & SW & NM & NE & \\
\hline bw-all & S03 & S17 & S04 & S16 & S12 & S15 & S01 & S05 & S18 & S07 & S13 & S10 & S08 & S14 & S11 & S02 & S09 & S06 & Section 4.3 \\
& SW & Eu & MW & Eu & NE & Eu & NE & SM & As & SW & NM & NW & MM & NE & NM & SM & NE & SE & & \\
\hline rtt-uS & S11 & S04 & S12 & S05 & S14 & S07 & S06 & S10 & S01 & S03 & S08 & S09 & S02 & S13 & & & & Section 4.4.2 \\
& NM & MW & NE & SM & NE & SW & SE & NW & NE & SW & MM & NE & SM & NM & & & & \\
\hline rtt-20 & S04 & S12 & S18 & S17 & S16 & S07 & S06 & S15 & S10 & S01 & S03 & S05 & S14 & S08 & S02 & S09 & S11 & S13 & Section 4.4.3 \\
& MW & NE & AS & Eu & Eu & SW & SE & Eu & NW & NE & SW & SM & NE & MM & SM & NE & NM & NM & \\
\hline
\end{tabular}

NE: US northeast, NW: US northwest, SE: US southeast, SW: US southwest, ME: US middle-east, MW: US middle-west MM: US middle-middle, Eu: Europe, As: Asia

includes the results from the greedy algorithm when considering all destinations. The row "bw-all" in Table 2 lists the replica selection sequence from the greedy algorithm, and the marginal utility from each replica. We can see that the bandwidth improvement spreads almost uniformly in the large range [5Mbps, $50 \mathrm{Mbps}]$. If using $5 \%$ as the threshold for marginal utility, only the first two replicas selected significantly contribute to the bandwidth performance improvement. From the row "bw-all" in Table 2, we note that geographic diversity does not play an important role. All these observations are very different from the results for RTT optimization.

\subsection{Variants of RTT Optimization}

In this section, we briefly present the results from three variants of the RTT optimization problem discussed in Section 4.2.

\subsubsection{Weighting Destinations Using Web Server Load}

So far we have assumed that clients from different prefixes have equal probability to request service, but this is not the case for many Internet services. To consider non-uniform client distributions, we obtained client request logs from five popular Web sites in the US to weight the RTT measurements. That is, the raw RTT to each destination is weighted using the total amount of packets exchanged with the clients that share the same prefix with the destination in our measurements.

The statistics of these Web logs are listed in columns 2-4 in Table 3. We can see that the client population only covers $5 \sim 10 \%$ of the prefixes that we measured. To weight the RTT measurements, we first compute the number of packets sent from a Web site to each of its clients. We then cluster these clients based on their network prefixes, obtain the total number of packets exchanged with each prefix (denoted as $p k t_{i}$ for prefix $i$ ). If the RTT to prefix $i$ is measured as $r t t_{i}$, we use $\left(r t t_{i} \times p k t_{i}\right)$ as the input to the greedy algorithm. We do not consider those prefixes which do not have any clients in the Web logs. Bandwidth measurements are weighted in similar way.

Applying the greedy algorithm on the weighted RTT values shows that the first five replicas still dominate the RTT performance improvement. However, the replica selection sequences, as listed in the last column of Table 3 , are very different from those obtained when giving all prefixes equal weight. First, the replicas outside the US are less important. Second, for all five Web sites, the first four replicas are always from NE, NM, SM, SW/MW, i.e., evenly distributed in the US. This clearly indicates that the client distributions of these Web sites are biased toward US users.

\subsubsection{US Replicas Only}

To consider the scenarios where deploying a replica outside the US is hard, we look at the case where we only use the 14 replicas inside the US. Using the same method as that in Section 4.2, we obtain the results as shown in Figure 11 and the "rtt-us" row in Table 2. The dashed curve in the top graph of Figure 11 is the RTT performance when using all 18 replicas; it is copied from Figure 8 for comparison. We can see that only $55 \%$ of paths have RTT less than $50 \mathrm{~ms}$ when using the US-only replicas, which is $13 \%$ worse than that when using all 18 replicas. Second, comparing with the brute-force solutions obtained in Section 4.2, the performance from the first four replicas selected here is $25 \%$ worse than the greedy-algorithm solution when using all replicas. These two results quantify the importance of replicas in Europe and East-Asia for RTT optimization. Finally, with the US-only replicas, only the first three replicas have marginal utilities larger than 5\%, and they are from the middle, the east coast, and the west coast of the US, which again highlights the importance of diverse geographic loca- 
Table 3. Replica selection sequence weighted by Web server load (measured in $\mathbf{4 7}$ hours)

\begin{tabular}{|c||r|r|r||l|}
\hline & $\begin{array}{r}\text { Number of } \\
\text { prefixes }\end{array}$ & $\begin{array}{r}\text { Load } \\
\text { (byte) }\end{array}$ & $\begin{array}{r}\text { Load } \\
(\mathrm{pkt})\end{array}$ & RTT \\
\hline \hline Web1 & 9759 & $235 \mathrm{G}$ & $369 \mathrm{M}$ & S14 S05 S07 S11 S06 S17 S04 S01 S12 S10 S03 S08 S15 S09 S18 S02 S16 S13 \\
\hline Web2 & 16412 & $163 \mathrm{G}$ & $337 \mathrm{M}$ & S14 S07 S11 S05 S15 S04 S18 S06 S01 S10 S16 S08 S12 S03 S09 S17 S02 S13 \\
\hline Web3 & 16244 & $157 \mathrm{G}$ & $182 \mathrm{M}$ & S11 S14 S04 S05 S01 S07 S06 S17 S10 S08 S02 S18 S12 S09 S16 S13 S03 S15 \\
\hline Web4 & 8113 & $23 \mathrm{G}$ & $203 \mathrm{M}$ & S14 S05 S04 S11 S06 S17 S07 S01 S08 S12 S10 S16 S02 S13 S18 S09 S03 S15 \\
\hline Web5 & 17484 & $124 \mathrm{G}$ & $157 \mathrm{M}$ & S14 S07 S11 S05 S17 S06 S04 S12 S18 S10 S01 S15 S08 S16 S03 S02 S09 S13 \\
\hline
\end{tabular}
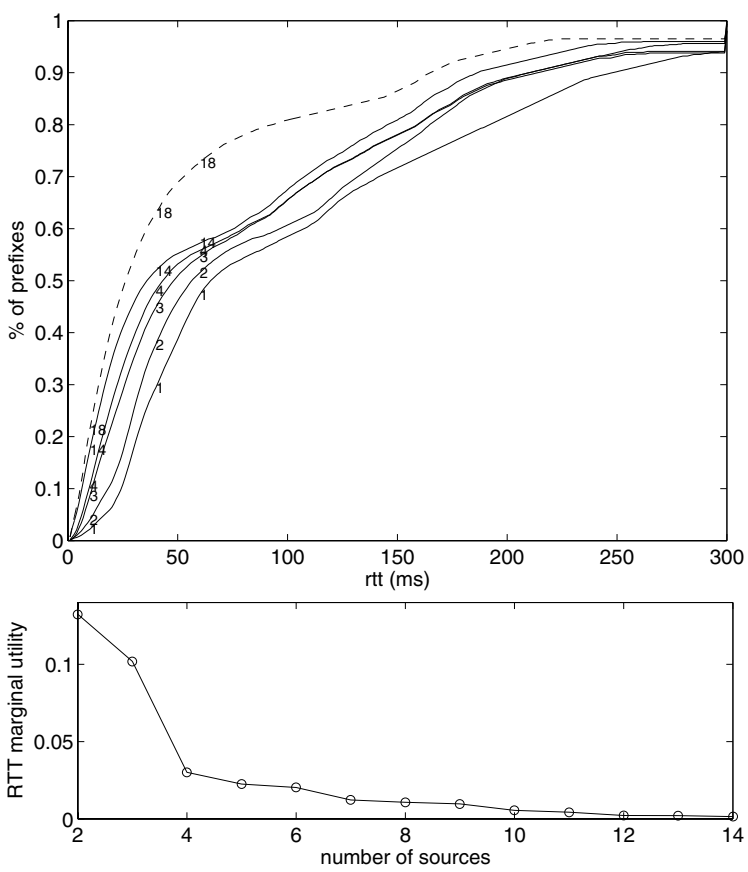

Figure 11. RTT optimization using only replicas in the US.

tions for RTT optimization.

\subsubsection{Optimizing The Worst Performance}

A previous study [12] has pointed out that CDNs can improve performance by simply avoiding directing clients to the worst replica. Based on this observations, we look at how well the infrastructure works if we focusing on improving the clients that have the worst performance. Specifically, we compute rtt_sum in such a way that only the worst $20 \%$ of the destinations are included, and then select the replica that can optimize this metric. The "rtt-20" row in Table 2 lists the replica selection sequences. The main difference with the results that consider all destinations is that there is one more European node in the top five replicas. Compared with the brute-force solution obtained in Section 4.2, the performance of the first four replicas is only $1.3 \%$ worse than that of the optimal four-replica set. Overall, we did not see a major improvement compared with the case where all destinations are considered.

\section{Performance of Applications}

The analysis in the previous section has shown that replicas can significantly improve RTT performance, but have much less impact on bandwidth performance due to bottleneck sharing. However, the performance of network applications is determined by data transmission time, which depends not only on RTT and available bandwidth, but also on data size. In this section, we consider these three factors to study the benefit of using replicas to reduce data transmission time. Below we first provide a simplified TCP model to characterize data transmission time as a function of available bandwidth, RTT, and data size. We then look at the transmission-time distribution for different data sizes with different number of replicas.

\subsection{Simplified TCP Throughput Model}

Simply speaking, TCP data transmission includes two phases: slow-start and congestion avoidance [9]. During slow-start, the sending rate doubles every roundtrip time, because the congestion window exponentially increases. During congestion avoidance, the sending rate and the congestion window only increase linearly. These two algorithms, together with the packet loss rate, can be used to derive an accurate TCP throughput model [20]. However, we can not use this model since we do not know the packet loss rate, which is very expensive to measure. Instead, we build a simplified TCP throughput model that only uses bandwidth and RTT information.

Our TCP throughput model is as follows. Let the path under considered have available bandwidth $a b w(\mathrm{Bps})$ and roundtrip time $r t t$ (second). Assume that the sender's TCP 
congestion window starts from 2 packets and that each packet is 1,500 bytes. The congestion window doubles every RTT until it is about to exceed the bandwidth-delay product of the path. After that, the sender sends data at a constant rate of $a b w$. This transmission algorithm is sketched in the code segment shown below. It computes the total transmission time $\left(t_{\text {total }}\right)$ for $x$ bytes of data along a path.

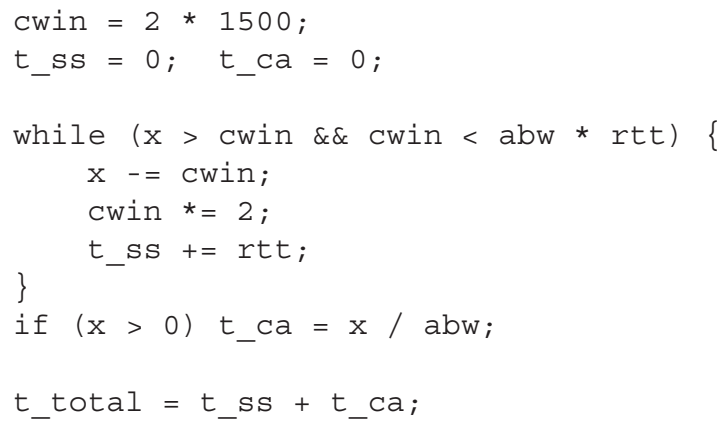

where $t_{-} s s$ and $t_{-} c a$ are the transmission time spent in the slow-start phase and the congestion avoidance phase, respectively. We say the data transmission is rtt-determined if $t_{-} c a=0$. We can easily derive the maximum rtt-determined data size as

$$
2^{\left\lfloor\log _{2}(a b w * r t t / 1500)\right\rfloor+1} * 1500(\text { byte })
$$

In the following, we call this size the slow-start size. Clearly, when the data is less than the slow-start size, it is rtt-determined.

This model ignores many important parameters that can affect TCP throughput, including packet loss rate and TCP timeout. However, the purpose of this analysis is not to compute an exact number, but rather to provide a guideline on the range of data sizes where RTT should be used as the optimization metric in replica hosting.

\subsection{Slow-Start Sizes and Transmission Times}

Using the above model, we compute the slow-start sizes for the 67,271 destinations for which Pathneck can obtain complete measurements. Figure 12 plots the distributions of slow-start sizes for the paths starting from each replica. Different replicas have fairly different performance; differences are as large as 30\%. Overall, at least $70 \%$ of paths have slow-start sizes larger than $10 \mathrm{~KB}, 40 \%$ larger than $100 \mathrm{~KB}$, and around $10 \%$ larger $1 \mathrm{MB}$. Given that web pages are generally less than $10 \mathrm{~KB}$, it is clear that their transmission performance is dominant by RTT and replica placement should minimize RTT. For data sizes larger than $1 \mathrm{MB}$, replica deployment should focus on improving bandwidth.

To obtain concrete transmission times for different data sizes, we use our TCP throughput model to compute data transmission time on each path for four data sizes: $10 \mathrm{~KB}$,

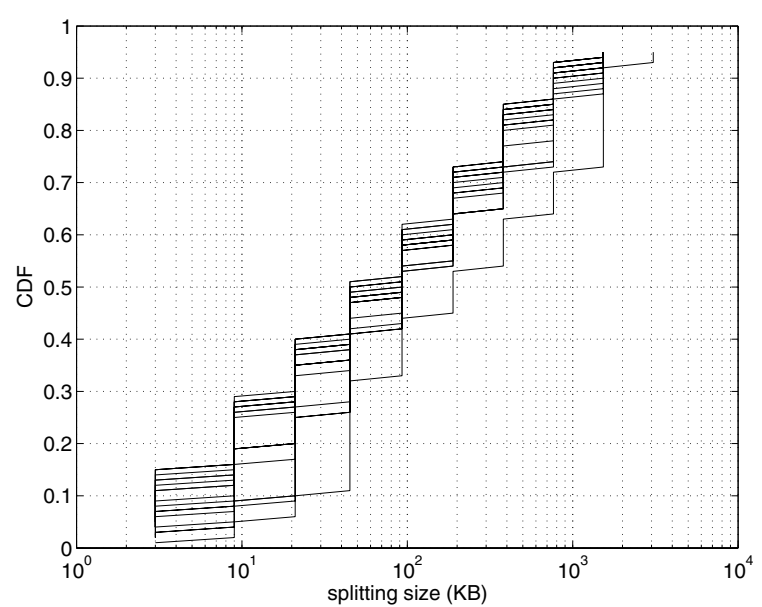

Figure 12. Cumulative distribution of the slow-start sizes
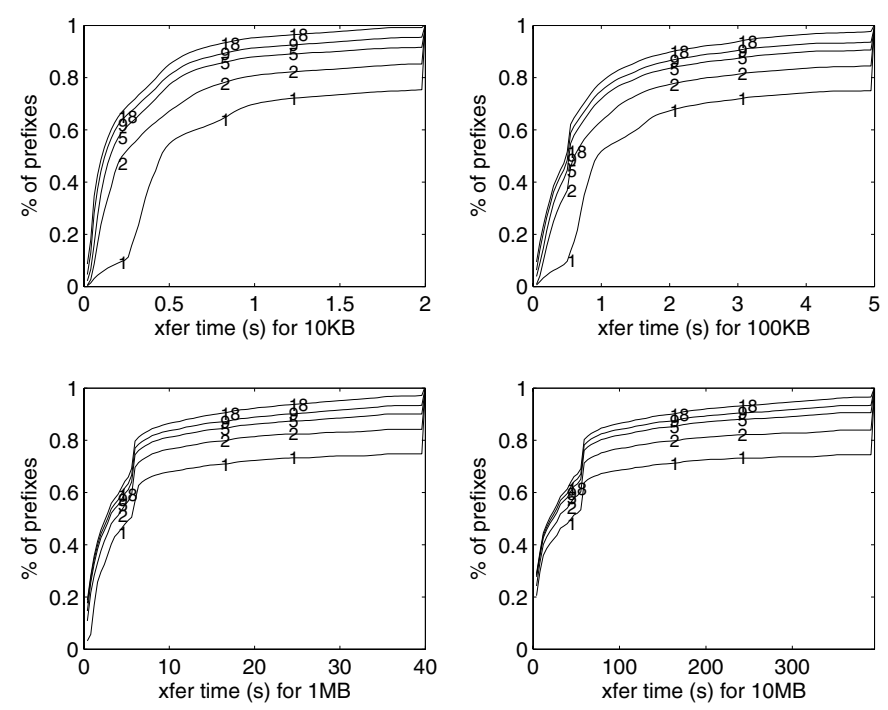

\section{Figure 13. Transmission times for different data sizes}

$100 \mathrm{~KB}, 1 \mathrm{MB}$, and 10MB. We then use the greedy algorithm to optimize data transmission times. The four subgraphs in Figure 13 illustrate the transmission-time distributions for each data size using different number of replicas. These figures are plotted the same way as that used in Figure 8. If we focus on the 80 percentile values when all 18 replicas are used, we can see the transmission times for $10 \mathrm{~KB}, 100 \mathrm{~KB}$, $1 \mathrm{MB}$ and $10 \mathrm{MB}$ are 0.4 second, 1.1 second, 6.4 second, and 59.2 second, respectively. These results are very useful for Internet-scale network applications to obtain an intuitive 
understanding about their data transmission performance.

\section{Related Work}

Content replication has been widely applied in real network systems [1,2]. The replication could be done for the whole mirror/proxy server, or for selected content objects. CDNs [22] generally belong to the former, while peer-topeer systems $[25,29]$ belong to the later. Our work is discussed in the context of server replication, but the results presented in this paper can also be applied for object replication.

The key question in content replication is how to deploy the replicas, including where to replicate and how many replicas should be used. Multiple solutions have been developed and two good overviews on these solutions can be found in [24, 14]. Different solutions often have different optimization goals, which include geographic location [19], network hops and latency [6, 23, 4, 18], retrieval costs $[17,16]$, update cost $[27,11]$, storage cost $[5,15]$, and QoS guarantee [26]. Many of the previous work considered two or three metrics together. For example, [27, 11] considered update cost and retrieval cost, $[5,15]$ considered storage cost and retrieval cost, while [13] considered three metrics: retrieval, update, and storage cost. More recently, [28] looks at the opportunities of optimizing a hosting service by dealing with network failures. To the best of our knowledge, no related work has studied the problem of optimizing bottleneck bandwidth performance. In addition, though propagation delay and transmission time have been considered by previous work, few of them considered these metrics in Internet-scale deployments. These are the two main differences between our work and the previous work, and they are also the main contributions of this paper.

In our work, we show that the performance gain becomes minimal after a number of replicas have been deployed. This is consistent with the observation in [10]. Our work is distinguished from [10] in the sense that we also study the geographic factor on replica placement and provide guidance based on the size of content objects.

Independent of the metrics used to optimize content distribution, greedy algorithms can often provide good solutions. The greedy algorithm proposed in our paper has near optimal solution. This is also consistent with the conclusion in [21], which compared several heuristics and found that a simple greedy algorithm works best. Greedy algorithms are also used in [26], which developed both "Greedy-Insert" and "Greedy-Delete" algorithms to solve QoS-aware placement problems.

\section{Conclusion}

In this paper, we use a set of large scale Internet measurements from 18 geographically diverse measurement sources to study how to optimize RTT and bandwidth performance in a content replication system. This set of measurements reveal for the first time the distribution of end-user access bandwidth. Based on a greedy algorithm, we show that only five carefully selected replicas are needed to optimize RTT performance in our infrastructure, while only two replicas are needed to optimize bandwidth performance. Moreover, the replicas selected for RTT optimization have a very clear geographic distribution, which is not the case for bandwidth optimization. Using real Web site logs, we also investigate the impact of Web client distribution on RTT performance optimization. We observe that the results are significantly different from those without considering such factor. Furthermore, we use a simplified TCP model to show that when the dominant data size of a network service is less than $10 \mathrm{~KB}$, replica deployment should optimize RTT; when the dominant data size is larger than $1 \mathrm{MB}$, replica deployment should optimize bandwidth.

\section{Acknowledgments}

Ningning Hu and Peter Steenkiste were in part supported by the NSF under award number CCR-0205266.

\section{References}

[1] Akamai. http://www.akamai.com.

[2] Digital Island. http://www.digitalisland.com.

[3] The Internet health report. http://www1.internetpulse.net.

[4] R. Carter and M. Crovella. Dynamic server selection using bandwidth probing in wide-area networks. In Proc. IEEE INFOCOM, 1997.

[5] I. Cidon, S. Kutten, and R. Soffer. Optimal allocation of electronic content. In Proc. IEEE INFOCOM, April 2001.

[6] S. G. Dykes, K. A. Robbins, and C. L. Jeffery. An empirical evaluation of client-side server selection algorithms. In Proc. IEEE INFOCOM, 2000.

[7] P. Francis, S. Jamin, C. Jin, Y. Jin, D. Raz, Y. Shavitt, and L. Zhang. Idmaps: A global Internet host distance estimation service. IEEE/ACM Transactions on Networking, October 2001.

[8] N. Hu, L. Li, Z. Mao, P. Steenkiste, and J. Wang. Locating Internet bottlenecks: Algorithms, measurements, and implications. In Proc. ACM SIGCOMM, August 2004.

[9] V. Jacobson. Congestion avoidance and control. In Proc. ACM SIGCOMM, August 1988.

[10] S. Jamin, C. Jin, A. R. Kurc, D. Raz, and Y. Shavitt. Constrained mirror placement on the internet. In Proc. IEEE INFOCOM, April 2001. 
[11] X. Jia, D. Li, X. Hu, W. Wu, and D. Du. Placement of webserver proxies with consideration of read and update operations on the internet. The Computer Journal, 46(4), July 2003.

[12] K. Johnson, J. Carr, M. Day, and M. Kaashoek. The measured performance of content distribution networks. In Proc. of the 5th International Web Caching and Content Delivery Workshop, May 2000.

[13] K. Kalpakis, K. Dasgupta, and O. Wolfson. Optimal placement of replicas in trees with read, write, and storage costs. IEEE Transactions on Parallel and Distributed Systems, 12(6):628-637, June 2001.

[14] M. Karlsson, M. Mahalingam, and C. Karamanolis. A framework for evaluating replica placement algorithms. Technical Report HPL-2002-219, HP Laboratories.

[15] M. R. Korupolu and M. Dahlin. Coordinated placement and replacement for large-scale distributed caches. IEEE Transactions on Knowledge and Data Engineering, 14(6):13171329, 2002.

[16] P. Krishnan, D. Raz, and Y. Shavitt. The cache location problem. IEEE/ACM Transactions on Networking, 8(5):568582, October 2000.

[17] B. Li, M. J. Golin, G. F. Italiano, X. Deng, and K. Sohraby. On the optimal placement of web proxies in the internet. In Proc. IEEE INFOCOM, April 1999.

[18] P. McManus. A passive system for server selection within mirrored resource environments using AS path length heuristics. http://pat.appliedtheory.com/bgpprox/.

[19] NetGeo - the Internet Geographic Database. http: //www. caida.org/Tools/NetGeo/.

[20] J. Padhye, V. Firoiu, D. Towsley, and J. Kurose. Modeling TCP throughput: A simple model and its empirical validation. In Proc. ACM SIGCOMM, September 1998.

[21] L. Qiu, V. N. Padmanabhan, and G. M. Voelker. On the placement of web server replicas. In Proc. IEEE INFOCOM, April 2001.

[22] M. Rabinovich and O. Spatscheck. Web Caching and Replication. Addison-Wesley, 2002.

[23] M. Sayal, Y. Breitbart, P. Scheuermann, and R. Vingralek. Selection algorithms for replicated web servers. In Proc. of the Workshop on Internet Server Performance, June 1998.

[24] S. Sivasubramanian, M. Szymaniak, G. Pierre, and M. van Steen. Replication for web hosting systems. ACM Comput. Surv., 36(3):291-334, 2004.

[25] I. Stoica, R. Morris, D. Karger, M. Kaashoek, and H. Balakrishnan. Chord: A scalable peer-to-peer lookup service for internet applications. In Proc. ACM SIGCOMM, August 2001.

[26] X. Tang and J. Xu. On replica placement for qos-aware content distribution. In Proc. IEEE INFOCOM, April 2004.

[27] J. Xu, B. Li, and D. L. Lee. Placement problems for transparent data replication proxy services. IEEE Journal on Selected Areas in Communications, 20(7):1383-1398, September 2002.

[28] M. Zhang, C. Zhang, V. Pai, L. Peterson, and R. Wang. Planetseer: Internet path failure monitoring and characterization in wide-area services. In Proc. OSDI, December 2004.

[29] B. Zhao, J. Kubiatowicz, and A. Joseph. Tapestry: An infrastructure for fault-tolerant wide-area location and routing. Technical Report UCB/CSD-01-1141, UCB. 